\title{
Immunological Assay of some Immobilizing Antigens of Paramecium aurelia, Variety 1
}

\author{
By J. O. BISHOP \\ Institute of Animal Genetics, University of Edinburgh
}

(Received 14 May 1962)

\begin{abstract}
SUMMARY
Quantitative methods are described for estimating the activity of antisera against Paramecium aurelia, variety 1, and purified preparations of the immobilizing antigen derived from the organisms. Confirmation was obtained that the relation between the serum concentration and the reciprocal of the immobilization time is approximately linear, for times between 3 and $10 \mathrm{~min}$. Titration of antigen preparations by a method based on inhibition of the immobilization reaction gave somewhat higher values than did titration by a gel-diffusion method. Cross-reactions between antigens controlled by genes at different loci and their various specific antisera were in all cases very small. The cross-reactions between antigens controlled by three genes at the $\mathrm{D}$ locus and their antisera varied according to the method used to measure them. Extensive cross-reactions were observed when the antisera were absorbed with heterologous paramecia and when precipitin formation in agar gel was followed. Only slight crossreactions were observed by two methods based on the immobilization reaction.
\end{abstract}

\section{INTRODUCTION}

The immunological specificity of the immobilization antigens of Paramecium aurelia is controlled by series of multiple alleles at several genetic loci (Sonneborn, 1951 ; Beale, 1952, 1954, 1957). In a given organism at a given time only the antigens controlled by genes at a single one of these loci can be detected, as a general rule. $\boldsymbol{P}$. aurelia is, however, a diploid organism and in a heterozygote two antigens controlled by different alleles may be detected simultaneously. A combination of environmental, cytoplasmic and genetic influences determines which of the genetic loci is expressed; by careful regulation of the conditions of culture it is possible to produce large clones in which nearly every organism expresses the same locus. When the specificities of two antigens are controlled by genes at different loci, the antigens are said to differ in 'type', and when specific antigen differences are controlled by alleles the antigens are said to differ in 'sub-type' (Beale, 1954, p. 113).

McClung (1943) showed that when a suspension of Paramecium was agitated without breaking the organisms, the supernatant fluid remaining after the animals were centrifuged off was capable of eliciting immobilizing antibody in the rabbit. Dr G. H. Beale (unpublished experiments) has shown that a water-soluble extract of homogenized Paramecium aurelia inhibits the immobilization of homologous organisms by immune serum; by using a technique of precipitin formation in agar gel Finger (1956) demonstrated that a water-soluble extract of $\boldsymbol{P}$. aurelia contained several antigens, one of which was the immobilization antigen. Preer (1959b) and 
Bishop (1961) developed methods of purifying the immobilization antigens, and showed that they were at least $95 \%$ protein in composition. A method of starch gel electrophoresis was used by Bishop \& Beale (1960) to measure the relative mobilities of several antigens. It was found that antigens of the same type were electrophoretically more similar than were antigens of different types. The present paper describes titration techniques used to assay immobilization antigens and antisera, and measurements of the cross-reactions between different antigens by these methods. These cross-reaction measurements are estimations of the immunological affinities of the antigens, and may be usefully compared with the electrophoretic observations.

\section{METHODS}

Stocks. The organisms were from stocks, 60, 90, and 103 of Paramecium aurelia, variety 1, maintained by Dr G. H. Beale. Serotypes D, G and S of stock 60 and serotype $D$ of stocks 90 and 103 were used.

Culture medium. A $7.5 \%(\mathrm{w} / \mathrm{v})$ suspension of dried grass was boiled for $15 \mathrm{~min}$., filtered, and the filtrate autoclaved $\left(20 \mathrm{~min}\right.$., $\left.120^{\circ}\right)$. Two days before use, it was diluted with $\mathbf{5 0}$ volumes of de-ionized water and seeded with a growing culture of Aerobacter aerogenes. The culture medium prepared in this way was mixed with an equal volume of a cleared Paramecium culture. When the new culture had itself cleared (i.e. when the paramecia had removed all the bacteria), half of the medium was removed and the organisms were harvested, while an equal volume of fresh culture medium was added to the remaining half.

Antisera. The paramecia were collected from cleared cultures by a modification of the electromigration method of Van Wagtendonk, Simonsen \& Zill (1952). The suspension was then centrifuged at $3000 \mathrm{~g}$ for $5 \mathrm{~min}$., resuspended at a concentration of $10^{6}$ organisms $/ \mathrm{ml}$. in solution $\mathrm{A}\left(0.013 \mathrm{M}-\mathrm{NaCl}, 0.003 \mathrm{M}-\mathrm{KCl}, 0.003 \mathrm{M}-\mathrm{CaCl}_{2}\right.$, 0.004 M-phosphate buffer, $\mathrm{pH} 6.8$ ) and homogenized by ejection from a hypodermic syringe. Rabbits (9 months old) were injected intravenously twice weekly for 3 weeks, $1 \mathrm{ml}$. of homogenate being given at each injection. Ten days after the last injection, $30 \mathrm{ml}$. of blood was taken from the marginal ear vein. The blood was allowed to clot, the serum drawn off, heated at $60^{\circ}$ for $30 \mathrm{~min}$. and dialysed overnight at $2^{\circ}$ against $3 \mathrm{l}$. of solution $\mathrm{A}$.

Antigen. Purified antigen was prepared as described elsewhere (Bishop, 1961). About $95 \%$ of the protein of purified antigen was immobilization antigen; carbohydrate and nucleic acid formed only about 2 and $0.5 \%$, respectively, of the total organic material.

Absorption of antisera by intact paramecia. The intact organisms, concentrated by electromigration, were centrifuged ( $3000 \mathrm{~g}$ for $5 \mathrm{~min}$.) and resuspended in the appropriate antiserum. The mixture was maintained at $32^{\circ}$ for $60 \mathrm{~min}$. and the organisms then centrifuged down.

Precipitin formation in agar. Antiserum, diluted with solution A, was mixed with an equal volume of $2 \%(w / v)$ agar (purified by the technique of Feinberg, 1956) and maintained at $60^{\circ}$. The mixture was pipetted into agar-coated capillary tubes (Oudin, 1952), inside diameter $2 \mathrm{~mm}$.; further steps were as described by Oudin (1952). Serial dilutions of purified antigen were pipetted on top of the agar layer, the tubes sealed with wax and incubated at $25^{\circ}$. At intervals of 1 day, the distance from the agar-antigen interface to the leading edge of the precipitin band was 
measured with a pair of dividers, and the equivalence value of antigen and antiserum was calculated as described by Oudin.

Estimation of immobilization time. Measurements of immobilization time were used in the assay of antiserum and in the assay of antigen. In the former case, a known volume (usually $0.05 \mathrm{ml}$.) of antiserum was pipetted into the three depressions of a depression slide. Next, the same volume of a suspension of paramecia (200 organisms $/ \mathrm{ml}$. solution $\mathbf{A}$ ) was added to each depression and a stopwatch was started. Each depression was examined at every 30 sec. under a binocular microscope, and the number of organisms not moving noted. The number which stopped in each $30 \mathrm{sec}$. period was later deduced from this cumulative record. The mean immobilization time was readily calculated for the organisms in each depression. The mean of means for the three depressions was the estimated immobilization time. When antigen was to be assayed the procedure was identical except that a small known volume of antigen in solution $\mathrm{A}$ was added to each depression after the antiserum, and $2 \mathrm{~min}$. before addition of organisms. A control slide was run in which an equal volume of solution $\mathbf{A}$ was added to each depression in place of the antigen.

Counting the organisms. The organisms were counted by making serial dilutions into solution $A$, and directly counting the number of organisms in ten $0.1 \mathrm{ml}$. samples of the final dilution.

\section{RESULTS}

\section{Estimation of the activity of antisera and solutions of antigen}

The immobilization of living paramecia by rabbit antiserum prepared against paramecia provides a ready means of estimating the immobilizing antibody content of different sera. According to the method of Sonneborn (1950) the immobilizing activity of an antiserum is measured by making a series of dilutions of the serum and finding the greatest dilution which will immobilize a small number of paramecia in the space of $2 \mathrm{hr}$. The same technique, with minor modifications, was used by Preer $(1959 a)$. It is, however, a very insensitive method in its present forms, as is any titration method which involves serial twofold dilutions.

Beale (1948) showed that the time taken by a given dilution of antiserum to immobilize paramecia (the immobilization time) was directly proportional to the dilution; that is, the greater the dilution, the greater the immobilization time. It seemed likely, therefore, that an accurate assay of antiserum activity could be developed, based on measurement of the immobilization time. To test the linearity of the dependence of immobilization time upon antiserum dilution, ten dilutions of $60 \mathrm{D}$ antiserum were made, each in triplicate, and the mean immobilization time of 30 homologous paramecia found for each of the thirty dilutions. When the reciprocal of the immobilization time $(1 / t)$ was plotted against the antiserum concentration $(A)$ it was seen that the line obtained was slightly but significantly curvilinear (Fig. 1). For the purpose of routine observations, this deviation from linearity was ignored, and the activity of an antiserum, in arbitrary units $(a) / \mathrm{ml}$., was taken as equal to $1 / t A$, where $t$ was the immobilization time in minutes and $A$ the fraction of the final reaction mixture constituted by undiluted antiserum. Where the purpose of the assay is to compare different antisera, the error introduced by the non-linearity of the relationship between antiserum concentration and time will be smallest when the difference in immobilization time is smallest. Accordingly serum dilutions which 
produced immobilization between 3 and $10 \mathrm{~min}$. after contact were used. The maximum error encountered, measured as the maximum deviation of the points from the straight line in Fig. 1, was then about $5 \%$, comparing favourably with the errors encountered with the older methods. Where more accurate determinations were desired, a series of dilutions of antiserum (calculated from a pilot experiment to give immobilization times of between 3 and $10 \mathrm{~min}$.) was made, and $a$ was calculated as the slope of the regression of $A$ upon $1 / t$.

This method has the advantages of great sensitivity and economy of material. It also provides a basis for the standardization of antisera produced against different types and subtypes of antigen, if it be assumed that the mechanism of immobilization is the same in each case. It suffers the disadvantage, however, of being dependent upon the physiological state of the paramecia used in the test. When using paramecia collected from cleared cultures by overnight electromigration into solution $\mathbf{A}$, the method gave highly reproducible results when the same antiserum was assayed on different occasions. However, paramecia collected directly by centrifugation from cleared, or uncleared, cultures were highly variable in their response to antiserum.

Antiserum concentration, $a$, as measured by this method, can be used to obtain an approximate value for the antiserum titre as defined by Sonneborn (1950) by taking $1 / 120 a$ as the titre. When this was done, the denominator was invariably 10-20\% greater than the denominator of the value obtained by direct titration by the Sonneborn method. This is doubtless related to the slight deviation from linearity observed in the relationship between antiserum concentration and the reciprocal of immobilization time (Fig. 1).

\section{Assay of antigen by the immobilization reaction}

Essentially the same reaction was used to assay solutions of antigen, by using the immobilization reaction to estimate how much antibody activity was lost upon contact with antigen. Figure 2 shows the relationship between the amount of antigen added (in arbitrary units) and the $\%$ of initial antibody activity lost, measured as $100\left(1-t_{0} / t\right)$, where $t$ and $t_{0}$ are the immobilization times in the presence and absence of antigen, respectively. Over the initial part of the curve, where the amount of added antigen is low compared with the amount of antibody, the slope is constant. The amount of antigen in a given solution, in units of antigen $/ \mathrm{ml}$. (g), can be calculated from $g=1 / G\left(1 / t_{0}-1 / t\right)$, where $G$ is the fraction of the final reaction mixture constituted by undiluted antigen solution. Units of antigen, g, so defined, are entirely equivalent to the units of antibody inactivated when antibody concentration is given by $a=1 / t A$ as described above. Measurements could be made only within certain limits of antigen and antibody concentration. To satisfy the conditions necessary for antiserum assay, of which this is a modification, times between 3 and $10 \mathrm{~min}$. had to be used. Secondly, to avoid the second part of the curve shown in Fig. 2, dilutions of antigen had to be chosen such that $t$ was less than $2 t_{0}$. In practice, a series of determinations was made within the range permitted by these two limitations and the value of $g$ was obtained as the slope of the regression of $-G$ upon $1 / t$.

The validity of this assay of the concentration of immobilizing antigen in solution depends upon the existence of linear relationships between antiserum concentration 
and immobilization time on the one hand, and between antigen concentration and amount of antibody inactivated on the other. The near linearity of the initial part of the curve of Fig. 2 is an indication of how closely these conditions are met over this range of concentration. It may be asked what relationship exists in this region between the amount of antigen added and the amount of antibody removed, in terms of molar ratios. The gel diffusion technique of Oudin (1952) provides a method of determining the equivalence concentrations of antibody and antigen solutions, and may readily be applied to the immobilizing antigen and antibody system. The same antigen can then be titrated against the same antiserum by the immobilization method. The concentration of antiserum equivalent to the given concentration of antigen under the conditions of the immobilization reaction is $A / G\left(1-t_{0} / t\right)$ where the symbols have the same meaning as before. Comparison of the values for the equivalence concentration of antiserum obtained by these two methods should reveal how close the immobilization method comes to measuring true immunological equivalence. Such tests were applied to solutions of purified antigen of two types, type $90 \mathrm{D}$ and type $60 \mathrm{D}$. The results (Table 1 ) show that the equivalence concentration of antiserum as measured by the immobilization method was $25 \%$ greater than the serological equivalence concentration, as measured by the gel-diffusion method. That is, a greater amount of antiserum was bound by a given amount of antigen as measured by the immobilization method. This suggests that the serological equivalence point may be found at the change of slope in Fig. 2, which represents a smaller antiserum to antigen ratio than does the initial slope. It may be noted (Table 1) that the true serological equivalencc concentrations of both serotypes examined bear the same relationship to the equivalence concentrations measured by the im. mobilization reaction.

Table 1. Equivalence concentrations of stock antisera against Paramecium aurelia, variety 1, for test antigen solutions, measured by the immobilization and gel-diffusion methods

The equivalence concentration of antiserum is given as the proportional concentration of the stock antiserum which is equivalent to the initial concentration of the test antigen.

Serotype of
antiserum
and
antigen
90D
60D

\begin{tabular}{|c|c|}
\hline \multicolumn{2}{|c|}{$\begin{array}{c}\text { Equivalence concentration } \\
\text { of antiserum }\end{array}$} \\
\hline $\begin{array}{l}\text { Immobilization } \\
\text { method }\end{array}$ & $\begin{array}{l}\text { Gel-diffusion } \\
\text { method }\end{array}$ \\
\hline $\begin{array}{l}0.0120 \\
0.0097\end{array}$ & $\begin{array}{l}0.0089 \\
0.0081\end{array}$ \\
\hline
\end{tabular}

Ratio of
immobilization
to gel-diffusion
equivalence
$\mathbf{1 \cdot 3}$
$\mathbf{1 . 2}$

Cross-reactions of antisera with heterologous antigens

Immunological cross-reactions of antisera with heterologous antigens were used as a measure of the structural similarities between the antigens. In particular, it was of interest to determine whether antigens of the same type but different sub-type differ from each other more than do antigens of different types. Previous work on the relationships between different immobilizing antigens of Parameciumaurelia, variety 1 (Beale, 1952), was based on a comparison of the titres obtained when a given antiserum was titrated against both homologous and non-homologous paramecia by the serial dilution method of Sonneborn (1950). Only very slight cross-reactions between 
antigens of different types were observed by this method. Within type $\mathbf{D}$ seven subtypes were detected (Beale, 1954) and several more sub-types have since been found (Dr G. H. Beale, unpublished data). Three of these, namely sub-types 60D, 90D and $103 \mathrm{D}$ were found to cross-react only to a very limited extent, to the same extent, in fact, as did the different types. These findings were re-examined by using the modified immobilization method described above, and were completely confirmed. The greatest extent of cross-reaction observed between any pair of antigens examined was $3.5 \%$.

Cross-reactions between antisera and heterologous antigens were also examined by investigating the inhibition of the immobilization reaction by heterologous antigen. For example, the extent to which the immobilization of $60 \mathrm{D}$ paramecia by $60 \mathrm{D}$ antiserum is inhibited by $60 \mathrm{G}$ antigen is a measure of the cross-reaction between $60 \mathrm{D}$ antiserum and $60 \mathrm{G}$ antigen. This method revealed no cross-reactions between different antigenic types and only very slight cross-reactions between the antigenic sub-types of type $\mathrm{D}$. In the latter case a slight cross-reaction was obtained at very high antigen concentrations; but the effects observed with heterologous antigen were unlike those observed with homologous antigen. The effect of adding homologous antigen in the immobilization reaction could not be distinguished from the effect of diluting the antiserum. On the other hand, when a high concentration of heterologous (different sub-type) antigen was added, in addition to a slight increase in immobilization time, an effect was observed which appeared very similar to the effect of adding a toxic concentration of saline. Addition of the heterologous antigen in the absence of antiserum did not produce such an effect. If the aberrant behaviour of the paramecia can be ignored, and only the immobilization time taken into consideration, cross-reactions between antigenic sub-types were always less than $10 \%$ of the homologous reaction when measured by this method.

A third useful method of studying the cross-reactivity of antisera with heterologous antigens was described by Finger (1957). Homologous or heterologous paramecia are incubated with the antiserum and then removed by centrifugation. The amount of antibody removed or neutralized by this means is measured by the decrease in antiserum titre which results, the antiserum being titrated against homologous paramecia by an immobilization method. The absorption of $90 \mathrm{D}$ antiserum by increasing amounts of $90 \mathrm{D}$ paramecia is illustrated in Fig. 3. The amount of antibody remaining after absorption for $1 \mathrm{hr}$. was measured by the immobilization method described in the present paper. When a sufficiently large number of paramecia was used in the absorption step (in this case about 12,000 paramecia/ml. antiserum) no residual immobilizing activity remained. When heterologous $60 \mathrm{D}$ paramecia (i.e. differing in sub-type) were used to absorb the antiserum, a point was reached (Fig. 3) at which an increase in the number of absorbing paramecia did not further the activity of the antiserum against homologous paramecium. The number decrease of heterologous paramecia $(60 \mathrm{D})$ required to reach this point was the same as the number of homologous paramecia required to exhaust the antiserum (in this case $12,000 / \mathrm{ml}$. antiserum). In the reciprocal experiment, namely the absorption of $60 \mathrm{D}$ antiserum by $60 \mathrm{D}$ and $90 \mathrm{D}$ paramecia, the same phenomenon was observed (Table 2). On the other hand, when antiserum was incubated with paramecia of a different type (as in the absorption of $60 \mathrm{D}$ antiserum by $60 \mathrm{G}$ paramecia) no significant decrease in titre was observed. These results are summarized in Table 2 , where 
the cross-reaction of each antiserum with heterologous paramecia is expressed as $\%$ decrease in'antiserum activity obtained with excess of absorbing paramecia. The crossreactions observed between the $\mathbf{D}$ sub-types by this method are thus greater by an order of magnitude than the cross-reactions observed by the immobilization methods.

Another method, more commonly used in immunological work, of quantitating cross-reactions, is the gel-diffusion method (Oudin, 1952). Where the same concentration of antigen is allowed to diffuse into a series of dilutions of the antiserum (mixed with agar gel) the equivalence concentration of the antiserum is found by extrapolating to the antiserum dilution which gives zero rate of migration of the

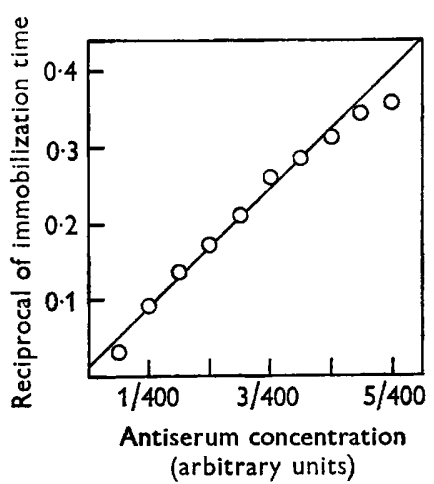

Fig. 1

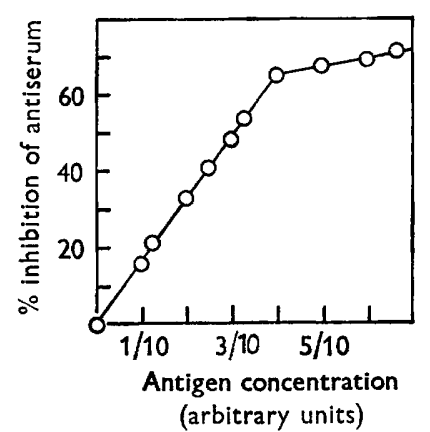

Fig. 2

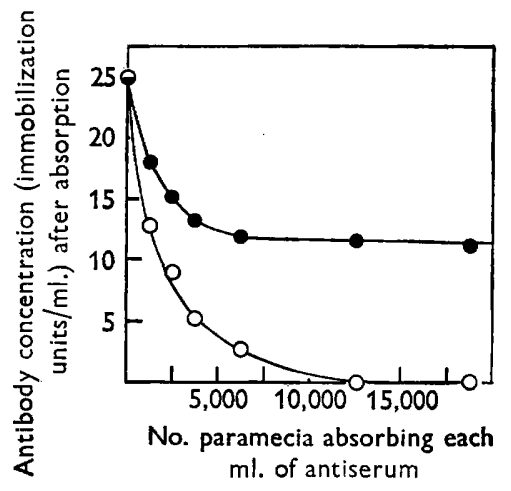

Fig. 3

Fig. 1. Dependence of the reciprocal of the immobilization time of Paramecium aurelia, variety 1 (serotype 60D), upon the antiserum concentration.

Fig. 2. Inhibition of the immobilization of Paramecium aurelia, variety 1 (serotype $60 \mathrm{D}$ ), by increasing concentrations of purified homologous antigen. The antiserum concentration in the untreated control was 0.32 immobilization units/ml. The percentage of immobilizing activity lost in the presence of antigen was calculated as described in the text.

Fig. 3. Loss of immobilizing activity from $90 \mathrm{D}$ antiserum upon incubation with increasing numbers of $90 \mathrm{D}(\mathrm{O})$ and $60 \mathrm{D}(\odot)$ paramecia.

Table 2. Cross-reactions between antisera against Paramecium aurelia, variety 1 , and immobilizing antigens measured by the serum-absorption and gel-diffusion methods

Cross-reactions are presented as \% of the value for the homologous reaction, as described in the text. A dash indicates that that particular cross-reaction was not measured.

\begin{tabular}{clrrrr}
\multirow{2}{*}{ Method } & Antiserum & 60D & $90 D$ & $103 D$ & $60 G$ \\
Serum absorption & anti-60D & 100 & 54 & - & 5 \\
& anti-90D & 52 & 100 & - & - \\
Gel-diffusion & anti-60G & 5 & 5 & - & 100 \\
& anti-60D & 100 & 47 & - & 5 \\
& anti-90D & 55 & 100 & - & 5 \\
& anti-103D & 39 & 60 & 100 & 5 \\
& anti-60G & 5 & 5 & - & 100 \\
& anti-60S & 5 & 5 & 5 & 5
\end{tabular}


precipitin band. The cross-reactivity of the immobilizing antigens and antisera was measured as follows. The equivalence concentrations of homologous and heterologous antisera for a given concentration of antigen were determined by parallel gel-diffusion experiments. The antisera were then titrated, each against its own homologous serotype paramecia, by the immobilization method. The equivalence concentration of each antiserum for the antigen used in the gel-diffusion experiments could then be expressed in terms of units of immobilizing activity. The percentage cross-reaction was calculated as the ratio of the homologous to the heterologous equivalence concentrations, multiplied by 100 . The immobilization method was thus utilized as a means of standardizing the concentrations of different serotype antisera. If the method is to be valid it is required that the mechanism of the immobilization reaction is the same for all serotypes. That this requirement is satisfied is indicated by: $(a)$ the relationship between the immobilization and geldiffusion titrations is the same for different antigens (Table 1); $(b)$ the immunological cross-reactions between the antigens as measured by this composite method are internally consistent, as will be seen below.

The results of the gel-diffusion cross-reaction measurements are presented in Table 2. In agreement with the cross-reactions measured by the immobilization methods, cross-reactions between different types of antigens and antisera were again very slight. In contrast to the immobilization results, but in agreement with the results obtained by absorbing antiserum with heterologous paramecia, the geldiffusion method showed strong cross-reactions between the three antigens controlled by allelic genes at the D-locus. It may be noted, too, that the cross-reaction of $60 \mathrm{D}$ antigen with $90 \mathrm{D}$ antiserum as measured by this method is quantitatively not significantly different from the cross-reaction of $90 \mathrm{D}$ antigen with $60 \mathrm{D}$ antiserum. The internal consistency of the method thus lends veracity to the assumption that the mechanism of immobilization is the same for different serotypes of Paramecium. Quantitatively, the cross-reaction between 60D antigen and 90D antiserum was greater than the cross-reaction between $60 \mathrm{D}$ antigen and $103 \mathrm{D}$ antiserum (Table 2). At the same time the cross-reaction between $90 \mathrm{D}$ antigen and $103 \mathrm{D}$ antiserum was greater than that between $90 \mathrm{D}$ antigen and $60 \mathrm{D}$ antiserum. Taken together, these results suggest the possibility that $90 \mathrm{D}$ antigen is an intermediate antigenic sub-type between sub-types $60 \mathrm{D}$ and $103 \mathrm{D}$.

\section{DISCUSSION}

An investigation of the electrophoretic mobilities of these antigens in starch gel (Bishop \& Beale, 1960) showed that antigens 60D, 60G and 60T (i.e. of different

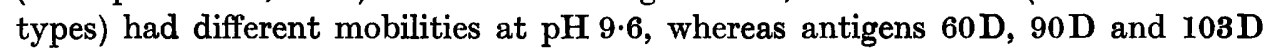
(i.e. sub-types of type $D$ ) had the same mobility. This agrees well with the immunological relationships revealed by the serum-absorption and gel-diffusion methods: antigens of different types showed negligible cross-reactions, while the three type $\mathrm{D}$ sub-types showed cross-reactions ranging from 25 to $50 \%$ of the homologous reactions. This, in turn, is not surprising in view of the genetic relationships of the antigens. It is to be expected that immobilizing antigens controlled by genes at different loci will differ from each other chemically and immunologically to a greater extent than will antigens controlled by allelic genes. 
Since the different antigenic types differ significantly in electrophoretic mobility, it may be supposed that the virtual absence between them of immunological crossreaction is a consequence of differences in primary structure. It is possible that the immunological differences between the type $\mathbf{D}$ sub-types are also consequent upon differences in primary structure, differences however which do not result in charge differences at pH 9.6 or which lead to charge differences too small to detect by the electrophoretic method employed.

The cross-reactions measured by the immobilization methods between the type D sub-types complicate the otherwise straightforward interpretation of the data. When antiserum was applied to heterologous paramecia (of different sub-types) little cross-reaction was observed as measured by the immobilization time of the paramecia. The heterologous paramecia were capable, however, of absorbing up to half of the immobilizing activity of the antiserum (Table 2). This observation, that paramecia absorb heterologous antiserum of different sub-type but are not readily immobilized by it, may be explained if it is supposed, simply, that the complex of antigen and heterologous antibody is a weak agent in inducing immobilization. This might occur, for example, if the complex were built up more slowly than the complex of antigen and homologous antibody. Since the immobilization method is a measurement of the rate of immobilization the heterologous antiserum would then give a weaker reaction.

Again, heterologous antigen, differing in sub-type, only slightly inhibited the immobilization of paramecia by homologous antiserum. Measured by the geldiffusion method, however, the heterologous antigen had up to half as much affinity for immobilizing antibody as did the homologous antigen. This effect, that although heterologous antigen combines with antibody it does not significantly prevent the antibody from causing immobilization, could result, for example, if homologous antigen were capable of displacing heterologous antigen from the antigen + antibody complex.

Although these explanations are very tentative, the basic difference between methods which show a high degree of cross-reaction and methods which show a low seems to be that the former measure the extent of complex formation between the heterologous antigen and antibody while the latter depend upon the extent of the immobilization reaction in the presence of such a complex. Similar discrepancies between different methods of titration have previously been reported by Finger (1956) and by Balbinder \& Preer (1959). In these cases, too, the greater crossreactions were observed when the extent of complex formation was measured. At this time, then, all indications point to the mechanism of immobilization as the source of such discrepancies.

This work was supported by a grant to Dr G. H. Beale from the Medical Research Council. I am indebted to Dr Beale for much helpful advice and encouragement.

\section{REFERENCES}

Balbinder, E. \& Preer, J. R. (1959). Gel diffusion studies on serotype and serotype transformation in Paramecium. J. gen. Microbiol. 21, 156.

BEALE, G. H. (1948). The process of transformation of antigenic type in $P$. aurelia, variety 4. Proc. nat. Acad. Sci., Wash. 34, 418.

BeAle, G. H. (1952). Antigen variation in P. aurelia, variety 1. Genetics, 37, 62. 
Beale, G. H. (1954). The genetics of Paramecium aurelia. Oxford University Press.

Beale, G. H. (1957). The antigen system of Paramecium aurelia. Int. Rev. Cytol. 6, 1.

Bishop, J. O. (1961). Purification of an immobilizing antigen of Paramecium aurelia, variety 1. Biochim. biophys. Acta, 50,471 .

Bishop, J. O. \& Beale, G. H. (1960). Genetical and biochemical studies of the immobilization antigens of Paramecium aurelia. Nature, Lond. 186, 734.

Feinberg, J. G. (1956). Agar clarification. Nature, Lond. 178, 1406.

Finger, I. (1956). Immobilizing and precipitating antigens of Paramecium. Biol. Bull., Woods Hole, 111, 358.

Finger, I. (1957). Immunological studies of the immobilization antigens of Paramecium aurelia, variety $2 . J$. gen. Microbiol. 16, 350.

McClung, L. S. (1943). A technique for the production of antisera for P. aurelia. J. Bact. 46, 576.

OudIN, J. (1952). Specific precipitation in gels and its application to immunochemical analysis. Meth. med. Res. 5, 335.

Preer, J. R. (1959a). Studies on the immobilization antigens of Paramecium. I. Assay methods. J. Immunol. 83, 276.

Preer, J. R. (1959b). Studies on the immobilization antigens of Paramecium. II. Isolation. J. Immunol. 83, 878.

SonNeborn, T. M. (1950). Methods in the general biology and genetics of $P$. aurelia. J. exp. Zool. 113, 87.

SonNEBorN, T. M. (1951). The role of the genes in cytoplasmic inheritance. In Genetics in the Twentieth Century. Ed. by I. C. Dunn. New York: The Macmillan Co.

Van Wagtendonk, W. J., Simonsen, D. H. \& Zili, L. P. (1952). The use of electromigration techniques in washing and concentrating cultures of Paramecium aurelia. Physiol. Zool. 25, 312. 